\title{
Creep Kinematics of Nonholonomic Systems
}

\author{
Jiunn-Cherng Wang, Han-Pang Huang \\ Department of Mechanical Engineering, National Taiwan University \\ Taipei, 10674 TAIWAN, R.O.C.
}

\begin{abstract}
Most studies about nonholonomic systems were developed under the absolute relation of constraint $e$ quations. Such ideally constrained systems are known somewhat relaxed in real world, which are claimed as complementarily constrained problems [12]. System$s$ with viscous frictions and/or flexibilities are typical cases at which the constraints are pseudo-violated rather than violated. These nonideal kinematics are treated in terms of nonholonomic creep behaviors, classified by two fundamental types : rotation creep and traverse creep. Creep coefficients are defined and proposed to illustrate the kinematical relations for such complementarily constrained systems. It can be shown that most nonholonomic vehicles can be described by both creep types, while the trailers attached to a car falls into the traverse type only. Therefore, we conclude the existing algorithms for nonholonomic motion planning and control should be modified as intrinsic creeps take place in nature. The creep notion can also be applied to the symmetry systems of conservation of angular momentum.
\end{abstract}

\section{Introduction}

Constrained systems of rolling without slipping or sliding without lateral components, such as the rolling disk, sleigh, and wheeled vehicles are typical examples of nonholonomic systems. The so-called "rolling without slipping" and "sliding without lateral components" mean mathematically the rolling or sliding is allowed only in the tangent space to a constraint manifold, and the constrained force is considered only in the normal space. The slipping or sliding in the normal space and the constrained force in the tangent space are artificially neglected.

The above ideally constrained problems are pseudorelaxed (or called pseudo-violated) in physical world. Since the viscous friction acts naturally at the contact regions (possibly point-, or line-, or surface-contact), the displacements in the normal space in those cases can be induced. Besides, the system flexibilities affect the constraints in an elastic manner. Under these nonideal sources, the original constraints will be modified into another ones. This pseudo-violation phenomenon is called a nonholonomic creep, which belongs to a complementarily constrained problem claimed in [12]. The term 'creep' originates from [11], and is different from the metallurgical creep of materials.

The realization of nonholonomic systems by viscous frictions was discussed in $[6,10]$. The conclusion in $[6]$ shows an ideally constrained motion can be obtained from the system free of constraints as the dissipative friction coefficient approaches infinity. Based on the same notion, this paper focuses on the kinematic analysis of the nonholonomic creep rather than the violated slipping. The latter is often accompanied by dry Coulomb frictions.

There are two fundamental types of creep found in most nonholonomic systems. One is rotation creep, the other is traverse creep. Both can be derived from specific forms of ideal constraints equations. The common motion of wheeled vehicles contains both types. Two creep coefficients are proposed to realize the pseudo-violated behaviors. Although the coefficients play similar role as those in $[3,4,13]$, they are different by definition.

Most algorithms for nonholonomic motion planning and control in [2,5,7-9] were based on the absolutely mathematical constraints. Observed from the kinematical simulation of a $2 \mathrm{D}$ creep disk, the motion gets mismatched. This mismatch can be compensated through visual feedback, such as driving a car. Most mobile robots, however, will incur imprecise performance due to using actuator signals only. Therefore, these existing algorithms should be modified to accommodate the creeps. Before the formal presentation, a motivative example from [10] is demonstrated to show the viscously deformed creep.

Example Consider the complementarily constrained motion of a pneumatically tired wheel shown schematically in Fig. 1. It is a top-view diagram of the front- 
right wheel of a car undergoing a right turn. The elliptic shadow, with center at $A$, depicts the contact region between the tire and the horizontal plane. The tire undergoes some deformation as a normal traction force $f$ acts on it through point $A$ and a centrifugal force $F$ through the tire center. Oxy means the horizontal plane and $O$ is the projection of the undeformed tire center. $x_{c}(<0)$ is the position of $A$ and $v$ is the wheel velocity.

In general, the creep equation can be expressed as

$$
\dot{x}_{c}=-k_{g} v x_{c}
$$

where $k_{g}$ is a growth parameter. Eq.(1) is not easy to apply; however, the small creep angle, defined as $\theta_{c} \triangleq \sin ^{-1} \dot{x}_{c} / v \approx \dot{x}_{c} / v$, can be used to illustrate such nonideal phenomenon. Rocard [11] related $f$ to $\theta_{c}$ as

$$
f=-k_{r} \theta_{c}
$$

with a resistance parameter $k_{r}$. Eq.(2) can explain the viscous creep where $f$ behaves proportional to the traverse velocity $\dot{x}_{c}$ in an opposite direction. Such behavior arises from the combined sources of flexibilities and viscous frictions.

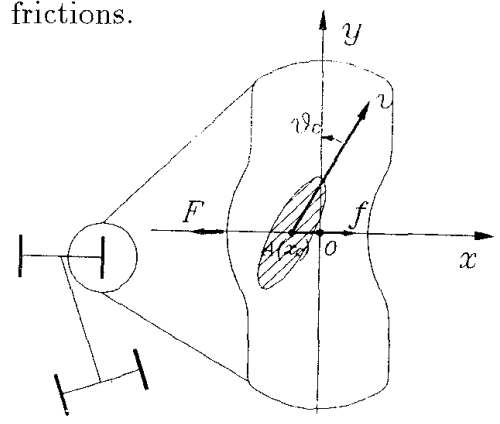

Fig. 1 Complementarily constrained creep of a pneumatically tired wheel.

\section{Rotation Creep Kinematics}

Consider the one-dimension disk rolling on a horizontal line, as shown in Fig.2. The pseudo-violation behavior falls into two cases: backward-creep $(0<$ $\left.e_{r c}<e_{r c 1}\right)$ and forward-creep $\left(e_{r c 2}<e_{r c}<0\right)$. $e_{r c}$ is called the coefficient of rotation creep defined later. $e_{r c 1}, e_{r c 2}$ are their corresponding limit values. The backward-creep occurs as the contact point moves instantaneously backward. This often takes place at the acceleration stage where the tractive friction force acts forwardly. The forward-creep behaves similarly. When $e_{r c}=0$, the rotation creep disappears and the rolling disk becomes an ideal disk.
Consider the disk with radius $r$. The disk center is located at $C$ and the contact point is at $A$ at the $t$ instant. Ideally, the instantaneous velocity of the disk at the contact point, $v_{A}$, vanishes. The ideal velocity $v_{i}$ and angular velocity $\omega_{i}$ of the disk satisfy

$$
v_{i}-r \omega_{i}=0 \text {. }
$$

As a matter of fact, the nonvanishing velocity $v_{A}$ results in (3) mismatched

$$
v_{l}-r w_{r}=v_{A}
$$

where $v_{l}$ and $\omega_{r}$ are real rolling velocities of disk.

Points $C$ and $A$ will move to $C^{\prime}$ and $A^{\prime}$ at the next time instant $t^{\prime}(=t+\Delta t)$. The net displacement and rotation angle are $d$ and $\theta\left(=\angle\left(A^{\prime} C^{\prime} A_{2}\right)\right)$, respectively. $A_{1}$ satisfies $\widehat{A^{\prime} A_{1}}=d$.

Definition The difference between $\theta$ and $\theta_{1}$ (= $\left.\angle\left(A^{\prime} C^{\prime} A_{1}\right)\right)$ is termed the angle of rotation creep,

$$
\theta_{r c} \triangleq \theta-\theta_{1}
$$

$\theta_{r c}$ is positive under the backward-creep and negative under the forward-creep. The rotation creep is denoted as type $\mathbf{R}$.

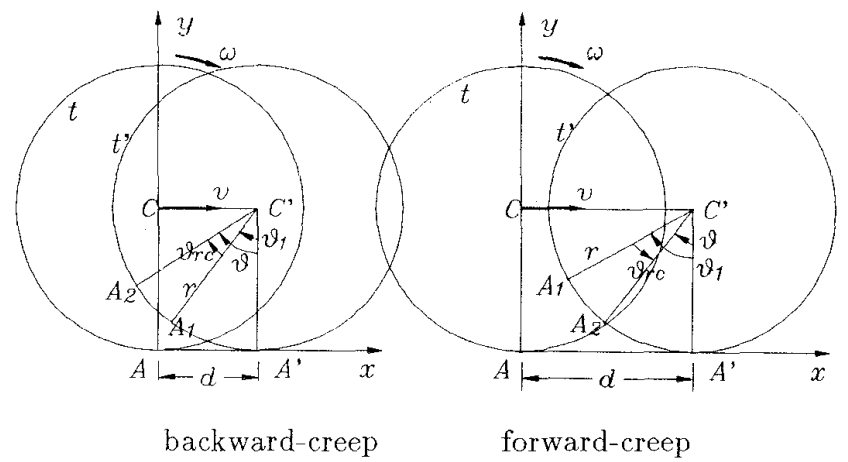

Fig. 2 Rotation creep of a 1D rolling disk.

During the interval $\Delta t$, the distance relation is

$$
d=r \theta\left(1-e_{r c}\right) .
$$

Definition The coefficient of rotation creep $e_{r c}$ is defined to illustrate the instantaneously kinematical mismatch between $d$ and $\theta$,

$$
e_{r c} \triangleq \lim _{\theta \rightarrow 0} \frac{\theta_{r c}}{\theta}
$$

Equivalently, $e_{r e}$ can also be defined through the velocity relation 


$$
e_{r c}=1-\frac{v_{l}}{r \omega_{r}}
$$

Note that $v_{l}$ and $\omega_{r}$ in (8) are not allowed vanishing simultaneously; i.e., the creep coefficient is not defined for the static disk. When $e_{r c}$ is a nonzero constant, $(d, \theta)$ or $\left(v_{l}, \omega_{r}\right)$ is called a rotational pair of creep motion according to [3].

Remark It is noticed that

- the definition of (7) or (8) acts different from the slipping coefficient $e_{s}$ in Ground Vehicles [13] and from the roll-slide number $\rho_{s}$ in the planar motion [4]. Their relations are

$$
\begin{aligned}
& e_{r c}=1-e_{s} \\
& e_{r c}=1-\frac{1}{\rho_{s}}
\end{aligned}
$$

where $e_{r c}$ is characterized by the complementarily constrained creep rather than the unconstrained slipping in $e_{s}$ and $\rho_{s}$.

- (4)-(8) are applicable for both the backward-creep and the forward-creep cases. Limit values $e_{r c 1}$ and $e_{r e 2}$ are constants in the vicinity of the origin. The slipping takes place as $e_{r c} \geq e_{r c 1}$ and the skidding as $e_{r e} \leq e_{r c 2}$.

- The mismatch between $d$ and $\theta$ or between $v_{l}$ and $\omega_{r}$ arises from the combined effects of disk's torsional flexibility and the viscous friction of tractive force with parameter $b_{R}$,

$$
f_{R}=-b_{R} v_{A}
$$

Fig. 3 depicts the principal stress curve of deformed radius lines under the torque $\tau_{R}$ and $f_{R}$. The real velocity of the point $A$ is $v_{A}=-r \omega_{r} e_{r c}$. Therefore, the rotation creep coefficient is related to the friction parameter as

$$
e_{r c}=\frac{f_{R}}{b_{R} r \omega_{r}}
$$

The measured $\omega_{r}$ and $f_{R}$ will give the value of $e_{r c}$ once $b_{R}$ is known, and in addition give the longitudinal velocity $v_{l}=\left(1-e_{r c}\right) r \omega_{r}$.

- Eq. (3) can be recognized as the restriction of displacement. Observe that (3) is simply equivalent to $d-r \theta=0$, and the fundamental type $\mathbf{R}$ creep can be obtained from a special form of the constraint,

$$
\dot{\pi}-\sigma \dot{q}=0
$$

where $\sigma$ is a constant and $\dot{\pi}, \dot{q}$ are respectively a quasivelocity and a generalized velocity. Many nonholonomic systems contain this type of constraints, in spite of the loss of physical senses for the chosen quasivelocities. The complementarily constrained problem of (13) can be often depicted as $\dot{\pi}-\sigma\left(e_{r c}\right) \dot{q}=0$, where $\sigma$ is a function of a suitably defined coefficient $e_{r e}$.

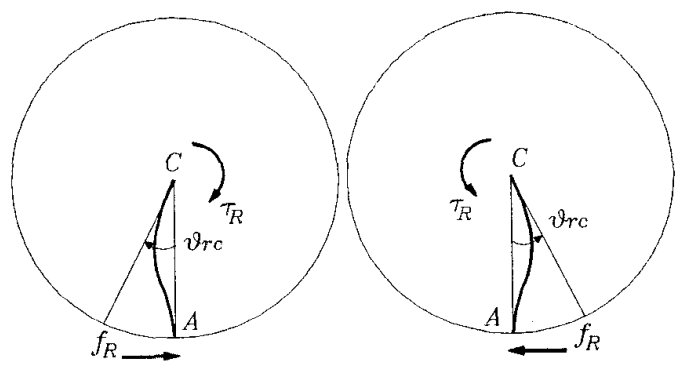

backward-creep forward-creep

Fig. 3 The horizontal torsion of flexible disk with deformed radius lines.

\section{Traverse Creep Kinematics}

Consider the Caplygin sleigh $[2,10]$ with the vertical coincidence of the center of mass $C$ and the contact point $A$. Two coordinate systems, the fixed $O x y z$ and the moving $A \xi \eta \zeta$, are used. The generalized coordinates are $(x, y, \phi)$, describing the position of $A$ and the orientation of sleigh. The ideal constraint equation is

$$
\dot{x} \sin \phi-\dot{y} \cos \phi=0 \text {. }
$$

Namely, the motion is allowed only along the principal $\xi$ axis.

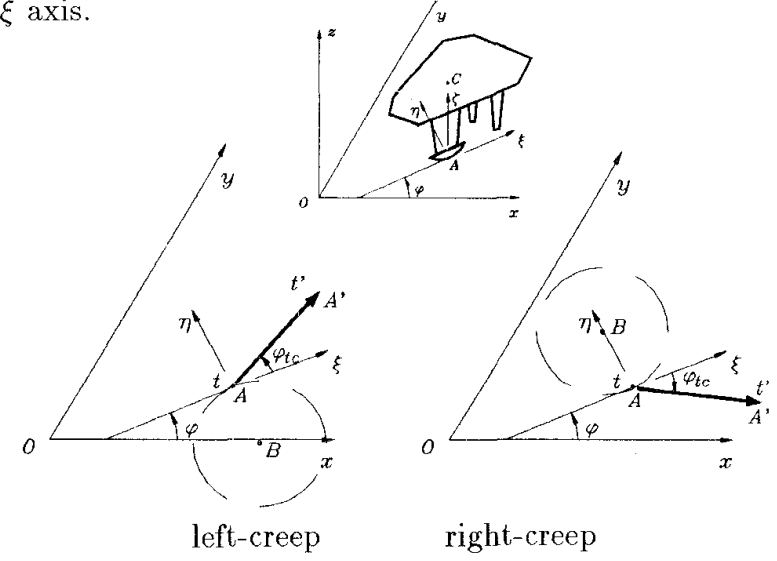

Fig. 4 Traverse creep of the Caplygin sleigh.

The pseudo-violation of the sleigh in Fig.3 can be categorized into two cases: left-creep $\left(0<e_{t c}<e_{t c 1}\right)$ 
and right-creep $\left(e_{t c 2}<e_{t c}<0\right)$. $e_{t c}$ is called the coefficient of traverse creep defined later. $e_{t c 1}, e_{t c 2}$ are their corresponding limit values. The left-creep occurs as the contact point $A$ moves instantaneously to left. This often takes place at the stage of clockwise-turn where the constrained friction force exerts to the right. The right-creep behaves similarly. When $e_{t c}=0$, the motion of sleigh is ideal.

The sleigh is located at $A$ at time $t$ with longitudinal velocity $v_{l}(=\dot{x} \cos \phi+\dot{y} \sin \phi)$ and angular velocity $\omega_{z}(=\dot{\phi})$. Physically, the sleigh moves along the dashed circle with the center at $B$ and the radius $v_{l} / \omega_{z}$. In fact, the traverse velocity $v_{t}(=$ $\dot{x} \sin \phi-\dot{y} \cos \phi)$ does not vanish in nature. Therefore, the sleigh will move to point $A^{\prime}$ at the next instant $t^{\prime}$.

Definition The angle between the ideal and real directions is called the angle of traverse creep,

$$
\phi_{t c} \triangleq \tan ^{-1} \frac{v_{t}}{v_{l}}
$$

$\phi_{t c}$ is positive under the clockwise left-creep and negative under the counterclockwise right-creep. The traverse creep is denoted as type $\mathbf{T}$.

Definition The coefficient of traverse creep is defined to illustrate the traversal motion in terms of the position of $A^{\prime}$ in the moving system $A \xi \eta \zeta$,

$$
e_{t c} \triangleq \lim _{\xi \rightarrow 0} \frac{\eta}{\xi}
$$

Equivalently, $e_{t c}$ can also be defined as

$$
e_{t c}=\frac{v_{t}}{v_{l}}\left(=\tan \phi_{t c}\right)
$$

where the static and purely orienting sleighs are excluded. $(\xi, \eta)$ or $\left(v_{l}, v_{t}\right)$ is termed a traverse pair of creep motion as $e_{t c}$ is a nonvanishing constant.

Remark It is noticed that

- the defined traverse creep angle $\phi_{t c}$ in (15) is similar to the side slip angle in [13].

- (15)-(17) can be applied to both the left-creep $\left(v_{t}, \phi_{t c}, e_{t c}>0\right)$ and the right-creep $\left(v_{t}, \phi_{t c}, e_{t c}<\right.$ $0)$. Besides, they are valid under the small working region $\left(e_{t c 2}, e_{t c 1}\right)$.

- Type $\mathbf{T}$ creep of sleigh can be brought from flexibility and/or from the viscous side force. The flexibility of bending causes the nonvertical coincidence of $C$ and $A$ under friction $f_{T}$ and centrifugal force $F$,

$$
f_{T}=-b_{T} v_{t}
$$

with parameter $b_{T}$. The vertical torsion gives direction misalignment under $\tau_{T}$ and $\bar{f}_{T}$ for surface contact. Both are shown in Fig. 5 and are consistent with Fig. 4. From (17) and (18), we find

$$
e_{t c}=\frac{-f_{T}}{b_{T} v_{l}}
$$

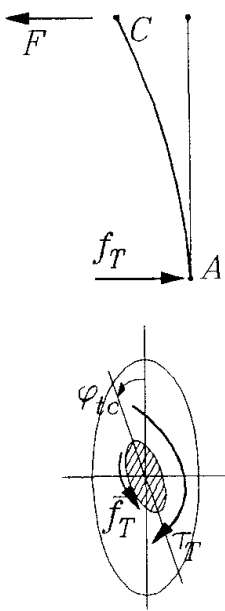

left-creep
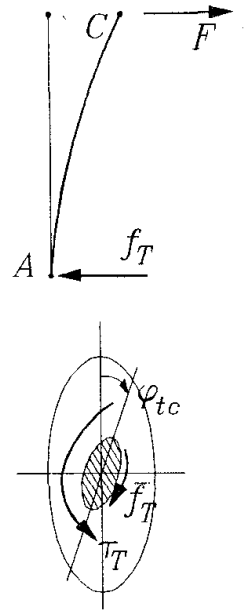

right-creep
Fig. 5 The bending and vertical-torsion flexibilities of sleigh.

- The ideally nonholonomic constraint in (14) has an intrinsic form in a $3 \mathrm{D}$ system,

$$
\dot{q}_{1}-\sigma\left(q_{2}\right) \dot{q}_{3}=0
$$

where $\sigma=q_{2}$ and $\left(q_{1}, q_{2}, q_{3}\right)=(x, \cot \phi, y)$ are generalized coordinates. (20) is known as the restriction of orientation $\left(=\dot{q}_{1} / \dot{q}_{3}\right)$ and is pseudorelaxed as $\dot{q}_{1}-\sigma\left(q_{2}, e_{t c}\right) \dot{q}_{3}=0$ with a suitable coefficient. Hence, the modified constraint of (14) becomes

$$
\dot{x}-\frac{\cot \phi-e_{t c}}{1+e_{t c} \cot \phi} \dot{y}=0 .
$$

\section{Nonholonomic Creep Kinematics of Wheeled Vehicles}

A rolling disk or a single wheeled vehicle on a $2 \mathrm{D}$ horizontal plane is depicted in Fig. 6 with four generalized coordinates $(x, y, \phi, \theta)$. The vehicle moves under two nonholonomic constraints,

$$
\dot{x} \cos \phi+\dot{y} \sin \phi-r \dot{\theta}=0
$$


car ( 7 coordinates, 3 holonomic constraints), the four wheeled car ( 8 coordinates, 4 holonomic constraints), and all wheeled cars with a single vehicle body. These conclude the theorem. Q.E.D.
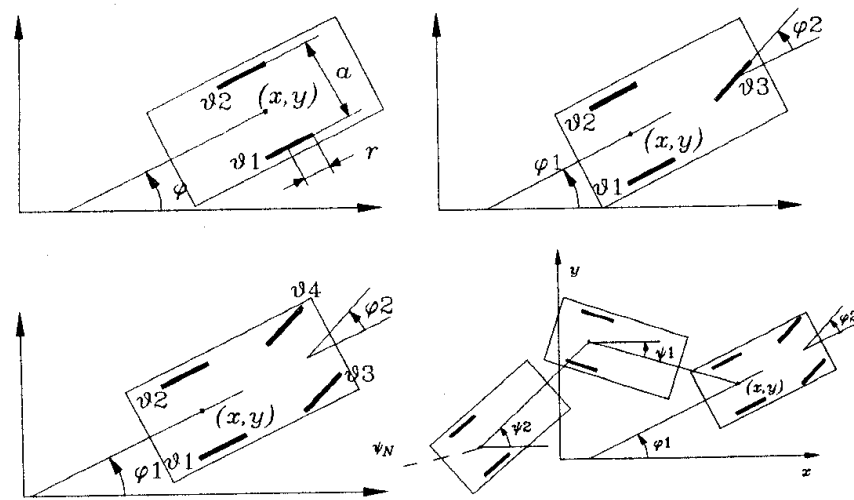

Fig. 8 Two, three, four wheeled vehicles and a car with $N$ trailers attached.

Theorem The nonholonomic creep of a car with $N$ trailers attached can be realized by the type $\mathbf{R T}^{N+1}$.

(proof) The configuration is shown in Fig. 8 with $4+N$ simplified coordinates $[8,9]$. Ideally, the aligned wheels of trailers roll and spin, but not slip traversely. The rotation slip or creep of $N$ trailers' wheels does not affect the whole motion since the distances between two hitches are fixed. Therefore, only traverse creep is needed for every trailers. The total numbers of coefficients of type $\mathbf{R}$ and type $\mathbf{T}$ to realize such system are 1 and $N+1$, respectively. Q.E.D.

The creeps of vehicles can be understood from the wheel flexibilities, including torsional and bending, and viscously tractive frictions. The above creep kinematics focus only on the nonholonomic systems with phenomenological constraints [1]. In fact, they are applicable to those of symmetry constraints, e.g., the hopping robot of conservation of angular momentum. Due to the viscous dissipation in air, similar coefficients of creep can also be defined.

\section{Conclusions}

Creep kinematics of nonholonomic systems are analyzed and classified into two fundamental types: the rotation and traverse creeps. Based on the natural viscous friction and/or system flexibilities, most wheeled vehicles contain both creep types which can be derived from a specific form of constraints. Since creep phenomena are essential features in nonholonomic systems, the algorithms of motion planning and control for nonholonomic systems should be modified. The development of creep dynamics and other issues are underway.

\section{Acknowledgment}

This work is partially supported by National Science Council, Taiwan R.O.C., under grant number NSC 81-0422-E-002-03.

\section{References}

[1] L. Bates, R. Cushman, J. Sniatycki, "Constraints and Reduction," Department of Mathematics and Statistics, Research Report, May, 1992.

[2] A.M. Bloch, M. Reyhanoglu, N.H. McClamroch, "Control and Stabilization of Nonholonomic Systems," IEEE Transaction on Automatic Control, Vol. 37, pp. 1746-1757, 1992.

[3] O. Bottema, "The $\lambda$-Pairs of Curves for a Cycloidal Motion," Mechanism and Machine Theory, Vol. 10, pp. 189-195, 1975.

[4] C.S. Cai, B. Roth, "On the Planar Motion of Rigid Bodies with Point Contact," Mechanism and Machine Theory, Vol. 21, No. 6, pp. 453-466, 1986.

[5] A.B.A. Cole, J.E. Hauser, S.S. Sastry, "Kinematic" $\mathrm{s}$ and Control of Multifingered Hands with Rolling Contact," IEEE Transaction on Automatic Control, Vol. 34, No. 4, pp. 398-404, 1989.

[6] A.V. Karapetian, "On Realizing Nonholonomic Constraints by Viscous Friction Forces and Celtic Stones Stability," PMM, U.S.S.R, Vol. 45, pp. 30-36, 1982.

[7] R. Mukherjee, D.P. Anderson, "Nonholonomic Motion Planning Using Stoke's Theorem," IEEE Intl. Conf. on Robotics and Automation, pp. 802-809, 1992.

[8] R.M. Murray, S.S. Sastry, "Nonholonomic Motion Planning : Steering Using Sinusoids," IEEE Transaction on Automatic Control, Vol. 38, No. 5, 1993.

[9] R.M. Murray, Z. Li, S.S. Sastry, A Mathematical Introduction to Robotic Manipulation, CRC Press, Boca Raton, 1994.

[10] J.I. Neimark, N.A. Fufaev, Dynamics of Nonholonomic Systems, American Mathematical Society, Providence, Rhode Island, 1972.

[11] Y. Rocard, Automobiles, Avionics, Point Suspendus, Masson, Paris, 1954; English transl., Ungar, New York, 1957.

[12] J.C. Wang, Geometry, Kinematics, Dynamics and Control for Complementarily Constrained Mechanical Systems, Ph. D. Dissertation (in preparation), Nat. Taiwan Univ., M.E., Taiwan, 1995.

[13] J.Y. Wong, Theory of Ground Vehicles, 2nd ed., John Wiley and Sons, New York, 1993. 


$$
\dot{x} \sin \phi-\dot{y} \cos \phi=0
$$

where $\dot{\theta}\left(=\omega_{r}\right)$ is the angular velocity of wheel rolling. (22) is similar to (13) with $\dot{\pi}=\dot{x} \cos \phi+\dot{y} \sin \phi, \sigma=r$, $\dot{q}=\dot{\theta}$. (23) acts as the ideal direction constraint of the sleigh in Section 3 .

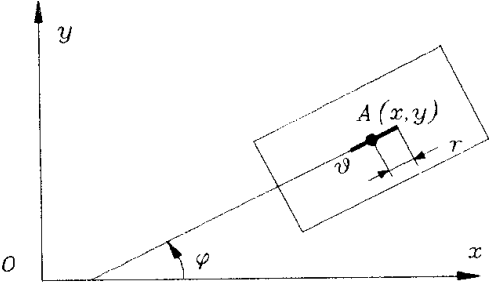

Fig. 6 A single wheeled car.

Proposition The pseudo-violated motion of the system (22) and (23) can be realized by the creep of type $\mathbf{R T}$; i.e., by both the rotation and traverse creeps.

If the coefficients $e_{r c}, e_{t c}$ are given, the nonholonomic creep motion can be calculated from the sensed $\omega_{r}$,

$$
\begin{gathered}
v_{l}=r \omega_{r}\left(1-e_{r c}\right) \\
v_{t}=r \omega_{r}\left(1-e_{r c}\right) e_{t c} .
\end{gathered}
$$

Then, $\dot{x}\left(=v_{l} \cos \phi-v_{t} \sin \phi\right)$ and $\dot{y}\left(=v_{l} \sin \phi+v_{t} \cos \phi\right)$ are integrable. Although the positional errors can be compensated by using vision feedback, the following simulation shows that a mobile robot with only actuator signals will incur considerable motion mismatch.

Simulation Consider the creep motion of a single wheeled vehicle with the following data : $r=0.5$ (meters $),\left.(x, y, \phi, \theta)\right|_{t=0}=(0,0,0,0),\left.(\dot{\phi}, \dot{\theta})\right|_{t=0}=(1,2)$ (radians/second), $\ddot{\theta}=-0.05$ (radians $/$ second $\left.{ }^{2}\right)$. The combined decelerated forward-creep and counterclockwise right-creep are kinematically simulated in $2 \pi$ seconds, assuming constant creep coefficients.

Four cases are simulated and shown by $x-y$ position in Fig. 7 : (i) $e_{r c}=-0.01, e_{t c}=-0.02$; (ii) $e_{r c}=-0.1, e_{t c}=-0.2$; (iii) $e_{r c}=-0.1, e_{t c}=-0.002$; (iv) $e_{r c}=-0.001, e_{t c}=-0.2$. The creep of type $\mathbf{R}$ dominates in the (iii) case, while the creep of type $\mathbf{T}$ dominates in the (iv) case.

The creep motions behave different from the ideal ones. Type $\mathbf{T}$ creep results in more positional difference than type $\mathbf{R}$ creep at the beginning stage since the latter causes mainly the mismatch of rolling angle and distance. This can be understood from the case (iii) where the rotated angle of creep motion is less than that of the ideal one, in spite of large traveling distance.
Due to energy conservation, the wheel's mass $m=$ $1(\mathrm{Kg})$, inertia $I=0.5\left(\mathrm{Kgm}^{2}\right)$, and polar inertia $J=1\left(\mathrm{Kgm}^{2}\right)$ are required to satisfy the rule, $m v^{2}+I \omega_{z}^{2}+J \omega^{2}=$ constant. For the kinematic simulation, however, the conservative creep is compared here with the ideal ones. The conservative and nonconservative creeps differ at the initial velocities in every integration steps. As to the real creep motion, the dynamics of wheeled vehicles should be taken into account.
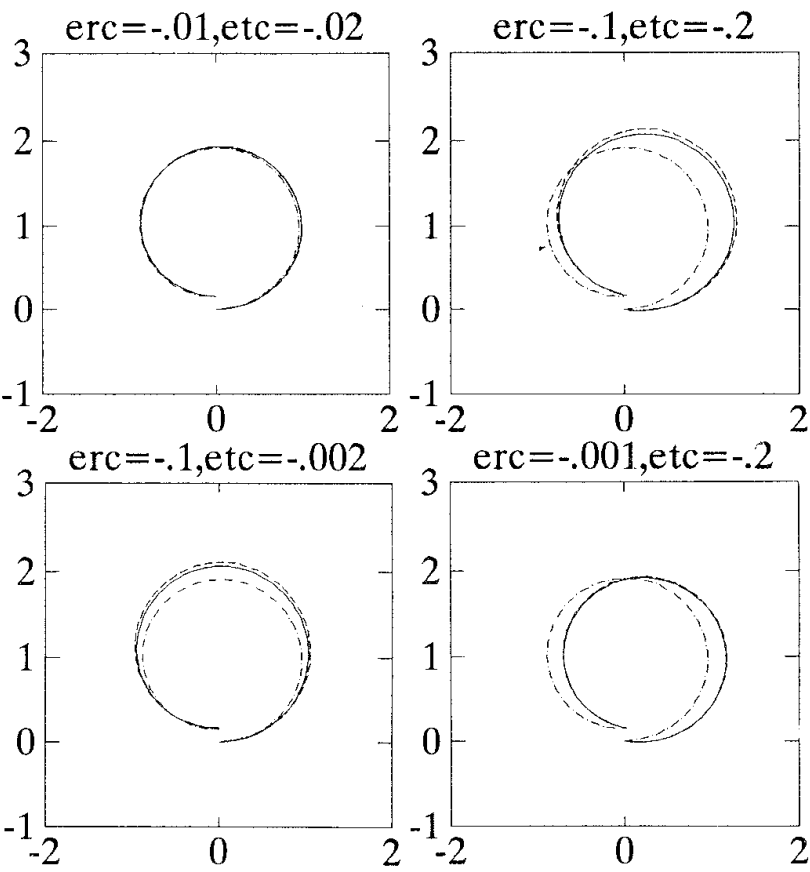

Fig. 7 Kinematical simulation of a single wheeled vehicle (solid: conservative creep, dashed: nonconservative creep, dashdot: ideal).

Theorem The nonholonomic creep of all wheeled cars with a single vehicle body can always be realized by the type of RT.

(proof by induction) Two, three and four wheeled cars are considered in Fig. 8. The configuration coordinates of a two wheeled car are $\left(x, y, \phi, \theta_{1}, \theta_{2}\right)$ with three constraints,

$$
\begin{gathered}
\dot{x} \cos \phi+\dot{y} \sin \phi-r\left(\dot{\theta}_{1}+\dot{\theta}_{2}\right) / 2=0 \\
\dot{x} \sin \phi-\dot{y} \cos \phi=0 \\
r\left(\theta_{1}-\theta_{2}\right)-a \phi=0
\end{gathered}
$$

where (28) is holonomic. Therefore, (26) and (27) will bring about the rotation and traverse creeps as mentioned in the Proposition. Similarly, only two nonholonomic constraints are found in the three wheeled 